\title{
TANDEM-X COMMISSIONING PHASE STATUS
}

\author{
Jaime Hueso Gonzalez, Markus Bachmann, Harald Hofmann
}

German Aerospace Center (DLR), Oberpfaffenhofen

\begin{abstract}
After the recent launch of the TanDEM-X satellite, the twin of TerraSAR-X, its demanding commissioning phase has started. On the one hand, it has to ensure the same monostatic operation performance as TerraSAR-X. On the other hand, the bistatic aspects have to be verified, which are essential for the acquisition of the global digital elevation model (DEM). This has to be done in a limited time period in order to keep the required nominal operation duration. This paper shows the summary of the commissioning phase activities and its running status.
\end{abstract}

Index Terms-TanDEM-X, TerraSAR-X, bistatic SAR, commissioning phase, formation flying

\section{INTRODUCTION}

TanDEM-X [1] is an Earth observation mission, result of the cooperation of the German Aerospace Center (DLR) and EADS Astrium GmbH, consisting of two twin synthetic aperture radar (SAR) satellites, TerraSAR-X (TSX) and TanDEM-X (TDX). TSX has been operative since 2007, and TDX has been launched on June 2010. Both satellites fly in a versatile, configurable and closely controlled helix orbit formation in order to acquire single-pass cross-track interferometric SAR (InSAR) images of the Earth with the optimal performance. The goal is to generate a global highly accurate digital elevation model (DEM) with $12 \mathrm{~m}$ resolution, $10 \mathrm{~m}$ absolute- and $2 \mathrm{~m}$ relative-height accuracy. This has to be achieved over the whole Earth's land surface before July 2014.

In order to assure the accurate completion of this DEM product, TDX has to undertake a precise commissioning phase in a tight time schedule. The commissioning phase activities range from the monostatic calibration (analogue to the one performed for TSX) to the commissioning of bistatic aspects like the innovative flight formation, the collision avoidance strategy, the upgraded mission planning and ground station network, the synchronization performance, the baseline calibration and the illumination risk handling.

The paper describes the status of this challenging commissioning phase and a detailed description of the main phases and activities.

\section{CHALLENGES}

\subsection{Mission Schedule}

The TSX satellite was launched in June 2007. Since its successful commissioning phase, where the instrument was accurately calibrated, TSX has remained very stable and reliable [2]. However, the warranted life time is limited. For acquiring the TanDEM-X DEM with the specified quality, at least two to three years of combined operation are required. Difficult terrain like mountainous areas needs to be acquired three or even four times to minimize shadowing and layover effects and phase unwrapping errors. In order to start as soon as possible with the nominal DEM acquisition, the TDX commissioning phase has to be completed in due time.

\subsection{Complexity}

The combination of the TerraSAR-X and TanDEM-X mission objectives increases the complexity of the system. Apart from acquiring the InSAR images for the DEM acquisition, the TerraSAR-X customers should not be disturbed. This is only possible by implementing a new mission planning concept to distribute the TerraSAR-X mission acquisition equally on both satellites. The SAR processing chain has also been extended to include an additional branch for interferometric processing [3], comprising bistatic and pursuit monostatic operation modes. The duration of the maintenance phases for activating the TanDEM-X functionalities have to be minimized.

\subsection{Collision Avoidance}

For the first time two satellites are operated at a nominal distance of a few hundred meters only. Safety is ensured by flying in a so-called "helix formation", where TSX and TDX have slightly different orbital planes [4].

\subsection{Exclusion Zones}

The helix formation results in one satellite turning around the other with one orbit periodicity. This means that there is a risk of mutual illumination during radar activities, when one satellite is in the direction of the main beam of the neighbor SAR antenna, which could cause damage to the electronic equipment. Therefore, a three-step fail-safe concept has been implemented to disable the transmission during this risky illumination periods. 


\subsection{TerraSAR-X Experience}

The experience gained during the commissioning and the more than two years of operation of TSX, together with its proven long term stability, allow us to face the TanDEM-X challenges with reasonable optimism.

\section{COMMISSIONING PHASE PLAN}

The commissioning phase has been divided into three main phases, as shown in Fig. 1.

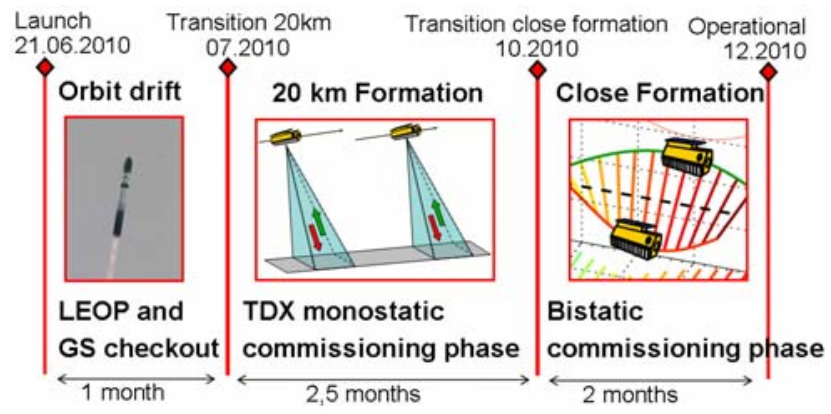

Figure 1: Three phases of the commissioning phase

The first phase lasts 30 days, starting with the Launch and Early Orbit Phase (LEOP) and ending with the drift process to position both satellites at $20 \mathrm{~km}$ along track distance. This phase includes the satellite checkout, the release of the antenna boom for data downlink and the test of the main spacecraft systems. In addition, all ground systems run through pre-defined checkout procedures. The goal in the launch is to position TDX in the correct reference orbit, but keeping a safe along track distance from TSX. After the LEOP, which takes approx. 7 days, TDX is slowly drifted towards TSX. The first phase concludes when the along track distance between the satellites is reduced and stabilized to $20 \mathrm{~km}$.

During the second phase, the monostatic commissioning phase, the system gets tuned for acquiring nominal TerraSAR-X-mission datatakes with TDX. This includes the instrument calibration and the product verification on the TDX as well as acquisitions in pursuit monostatic mode. The first bistatic test acquisitions will take place here, as well as specific interferometric experiments that require a high along track distance. It lasts around 2.5 months, and towards the end, the mean along track distance between the satellites is reduced to 0 and the helix formation with typical cross track distances of $200 \mathrm{~m}$ are set.

In this way, a close formation is achieved, which is the baseline for the third part of the commissioning phase, the bistatic one. Here, the first cross-track bistatic acquisitions are acquired, and the calibration, verification and validation of the bistatic aspects are performed. The bistatic commissioning phase has an estimated duration of 2 months, which means that the whole commissioning will last until December 2010.

At this point, the operational DEM acquisition will start, also in a close formation, but after scaling the helix formation parameters [1].

\section{ACTIVITIES AND PHASES}

\subsection{LEOP and Ground Segment Checkout}

The ground stations (satellite tracking) and their running systems (transcription and processing systems) have to be checked out. Also the mission planning system, which mainly comprises the verification of the power thermal model, the splitting of replays, parallel SAR acquisitions and the data dump and test of the left looking mode, has to undertake a checkout process. The verification of the orbit and attitude products should verify their required accuracy. Once this is working, the instrument will be activated for the first time. The first performance parameters of the instrument have to be analyzed and first initial acquisitions will be performed.

Finally, the complete chain from ordering to the generation of the processed image (see Fig. 2) has to be checked out.

\subsection{Monostatic Commissioning Phase}

Although during this phase the mean along track distance is $20 \mathrm{~km}$, a helix relative movement between the satellites is configured, similar to the one to be used in the close formation. In this way, the behavior of the formation is safely studied before eliminating the along track distance. The SAR instrument calibration is the main driver for the duration of this phase. A defined number of acquisitions

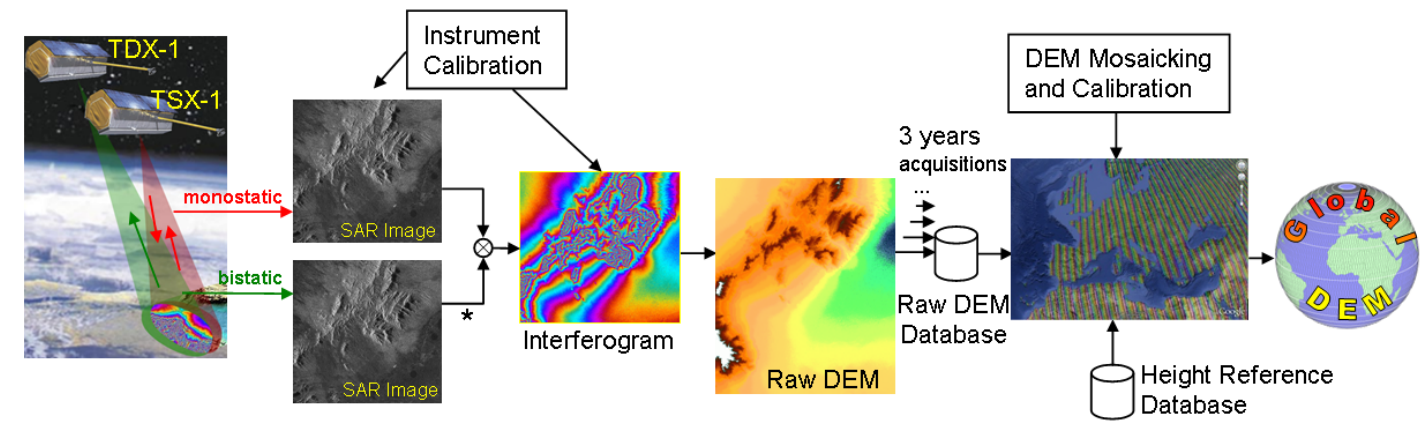

Figure 2: Simplified processing chain of the TanDEM-X global DEM 
over the ground targets at the DLR calibration field in southern Germany [2] are required for an accurate calibration. To achieve the same accuracy as obtained for TSX, two cycles for geometrical and pointing calibration are required. Using a precise antenna model, the absolute calibration can be reduced to only three cycles. The verification of the SAR performance is important to ensure the product quality. Here, the Noise Equivalent Sigma Zero (NESZ), the Signal-to-Noise Ratio (SNR) and the ambiguities are evaluated. These verifications are repeated during the bistatic phase. All SAR products have to be verified to assess the product quality and compliance with the specification.

\subsection{Bistatic Commissioning Phase}

The TanDEM-X mission has now the capability of acquiring cross-track bistatic SAR data. Parameters like the receiver gain, the echo window and the pulse repetition frequency have to be adjusted and optimized for this operational mode.

Since both satellites comprise independent local oscillators, the relative phase differences affect the height accuracy of the DEM. Therefore, synchronization between both oscillators is required. This is done by exchanging special synchronization pulses through the X-band horn antennas of a dedicated synchronization link. The performance of this synchronization link has to be verified with special datatakes.

The TanDEM-X interferometric processor has to be operationally verified, too. Its task is to generate the raw DEMs from the interferometric SAR images after the coregistration and phase unwrapping processes.

For the study of the systematic height errors in the DEM, caused by instrument and baseline errors [5], and the cancellation of potential offsets, a series of raw DEMs will be generated during this phase over chosen test sites over the world with suitable characteristics (see Fig. 3). Precise height references (laser DEMs of ICESat references [6]) are available over these test sites, which allow calculating the height error in these locations.

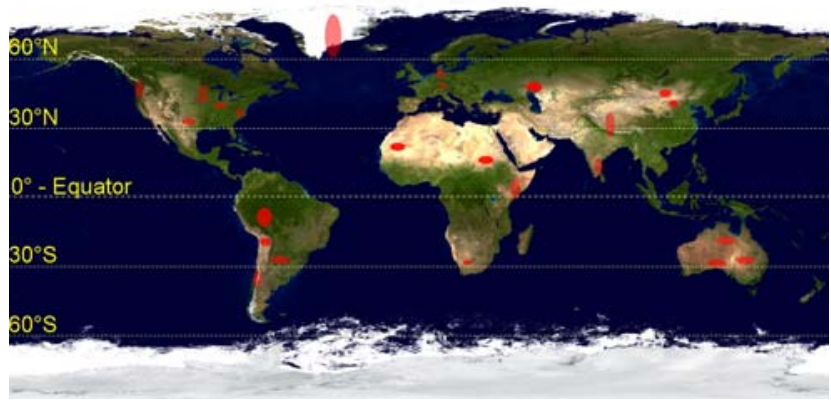

Figure 3: Distribution of the TanDEM-X test sites

The raw DEMs are then calibrated in height and mosaicked by the DEM calibration and mosaicking processor. For testing this processor, adjacent datatakes are acquired over certain of the test sites in Fig. 3, as well as crossing acquisitions. Adjacent acquisitions have to be acquired in consecutive repeat cycles, fact that sets a constraint in the bistatic commissioning phase duration.

\section{CONCLUSION}

The commissioning phase planning provides an overview on the tasks to be performed during the TanDEM-X commissioning phase. The main challenges are the tight schedule, the close formation flight and the extension of an operational running system. The three phases are packed with different verification tasks and calibration activities. However, with the experience gained with TSX and its high stability, a successful TanDEM-X commissioning phase is foreseen.

\section{ACKNOWLEDGMENT}

The authors want to explicitly thank all participants of the commissioning phase meetings at DLR for all productive discussions and the resulting commissioning phase plan.

The TanDEM-X project is partly funded by the German Federal Ministry for Economics and Technology (Förderkennzeichen 50 EE 0601).

\section{REFERENCES}

[1] G. Krieger, A. Moreira, H. Fiedler, I. Hajnsek, M. Werner, M. Younis, and M. Zink, "TanDEM-X: A Satellite Formation for High Resolution SAR Interferometry," IEEE Trans. on Geoscience and Remote Sensing, vol. 45, no. 11, pp. 3317-3341, Nov. 2007.

[2] M. Schwerdt, B. Bräutigam, M. Bachmann, B. Döring, D. Schrank and J. Hueso, "Final TerraSAR-X Calibration Results Based on Novel Efficient Methods," IEEE Trans. on Geoscience and Remote Sensing, vol. 48, no. 2, pp. 677-689, Feb 2010.

[3] T. Fritz, H. Breit, M. Eineder, N. Adam, and M. Lachaise, "Interferometric SAR Processing: From TerraSAR-X to TanDEM-X," European Conference on Synthetic Aperture Radar (EUSAR), Friedrichshafen, Germany, June 2008.

[4] O. Montenbruck, R. Kahle, S. D'Amico, and J.-S. Ardaens, "Navigation and Control of the TanDEM-X Formation," The Journal of the Astronautical Sciences, vol. 56, no. 3, pp. 341357, July-September 2008.

[5] J. Hueso, M. Bachmann, G. Krieger, and H. Fiedler, "Development of the TanDEM-X Calibration Concept: Analysis of Systematic Errors", IEEE Transactions on Geoscience and Remote Sensing (TGRS), vol. 48, no. 2, pp. 716-726, February 2010.

[6] J. B. Abshire, X. Sun, H. Riris, J. M. Sirota, J. F. McGarry, S. Palm, D. Yi, and P. Liiva, "Geoscience Laser Altimeter System (GLAS) on the ICESat Mission: On-orbit measurement performance," Geophysical Research Letters, vol. 32, Nov. 2005. 